OPEN ACCESS

Edited by:

Gang Zhang,

Nanjing Normal University, China

Reviewed by

Li-an Bian,

Changsha University of Science and

Technology, China

Xue Ren,

Shenzhen University, China

*Correspondence:

Kai-Da Xu

kaidaxu@ieee.org

Specialty section:

This article was submitted to

Radiation Detectors and Imaging,

a section of the journal

Frontiers in Physics

Received: 06 August 2021

Accepted: 16 August 2021

Published: 26 August 2021

Citation:

Tan L, Wang Q, Guo Y-J, Cui J and Xu K-D (2021) Bandpass Filter Based on Spoof Surface Plasmon Polaritons

With a Switchable High-Selectivity

Notch Band.

Front. Phys. 9:754510.

doi: 10.3389/fphy.2021.754510

\section{Bandpass Filter Based on Spoof Surface Plasmon Polaritons With a Switchable High-Selectivity Notch Band}

\author{
Longfei Tan ${ }^{1}$, Qiangji Wang ${ }^{2}$, Ying-Jiang Guo ${ }^{3}$, Jianlei Cui ${ }^{4}$ and Kai-Da Xu ${ }^{5 *}$ \\ ${ }^{1}$ Sichuan Fire Science and Technology Research Institute of Ministry of Emergency Management, Chengdu, China, ${ }^{2}$ Nanjing Ray- \\ Chip Microsystem Co. Limited, Nanjing, China, ${ }^{3}$ Microsystem and Terahertz Research Center, China Academy of Engineering \\ Physics, Chengdu, China, ${ }^{4}$ State Key Laboratory for Manufacturing Systems Engineering, Xi'an Jiaotong University, Xi'an, China, \\ ${ }^{5}$ School of Information and Communications Engineering, Xi'an Jiaotong University, Xi'an, China
}

A substrate integrated waveguide (SIM) based spoof surface plasmon polariton (SSPP) is proposed for the design of bandpass filter (BPF). The left and right edge cutoff frequencies of the passband can be easily adjusted by changing the parameters of SIW and ring slot embedded into the SIW. Then, four half-wavelength circular slots are added on two sides of the SSPP located at the center of the circuit to introduce a high-selectivity notch band. In order to make the notch band switchable, four full-wavelength circular slots and four PIN diodes are applied instead of the four half-wavelength circular slots. As the PIN diodes are under the ON state, the notch band will be generated within the passband of BPF. On the contrary, as the PIN diodes are under the OFF state, the notch band will disappear. To validate the design idea, two BPF examples are fabricated and measured, whose simulation and measurement results are both in reasonably good agreement.

Keywords: bandpass filter, spoof surface plasmon polaritons, substrate integrated waveguide, switchable function, high selectivity

\section{INTRODUCTION}

Surface plasmon polaritons (SPPs) are surface waves in the optical frequency band that propagate along the metal-dielectric interface but decay fast in the vertical direction [1]. In 2004, the concept of designer or spoof SPPs (SSPPs) was defined through the presentation of a periodic metal hole array structure that supports SPP-like mode with strong field confinement at microwave and terahertz frequencies [2]. From then on, the theory of SSPPs has been further improved [3], and the corresponding designs and applications have been further extended.

Numerous studies on SSPP-based devices and antennas have been presented in the recent decade [4-16], most of which are designed on the planar structures, such as SSPPs using PCB [5-13] and onchip process $[15,16]$. Utilizing the high-frequency cut-off characteristics of SSPP, the most common designs are SSPP based lowpass filters $[12,13]$. Moreover, many SSPP based bandpass filters (BPFs) have been also designed through adding the balun or other high-pass characteristic structures $[5,6]$.

However, most of these reported works lack of the actively dynamic adjustment ability. Due to the increasingly complicated environments with different interferences, it is urgently needed to study the reconfigurable or switchable devices based on SSPPs, which is of great significance for the development of plasmonic integrated circuits applications. Especially, BPFs with notch band are of importance and usefulness to suppress unwanted signals at the frequencies of interest. Consequently, some works on 

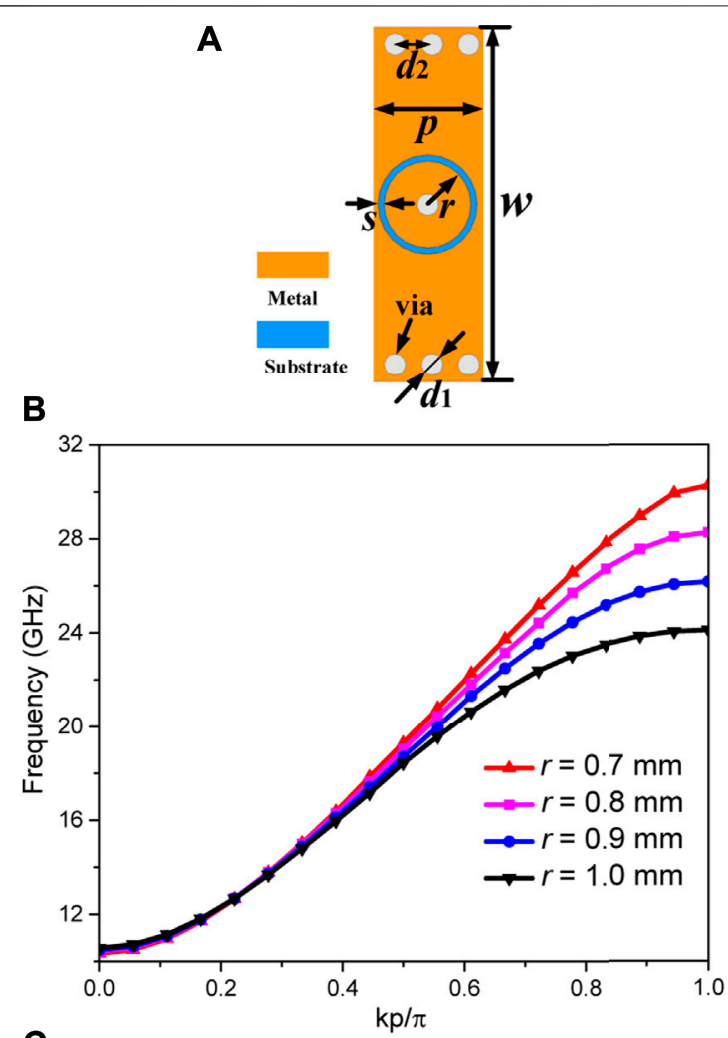

$c$

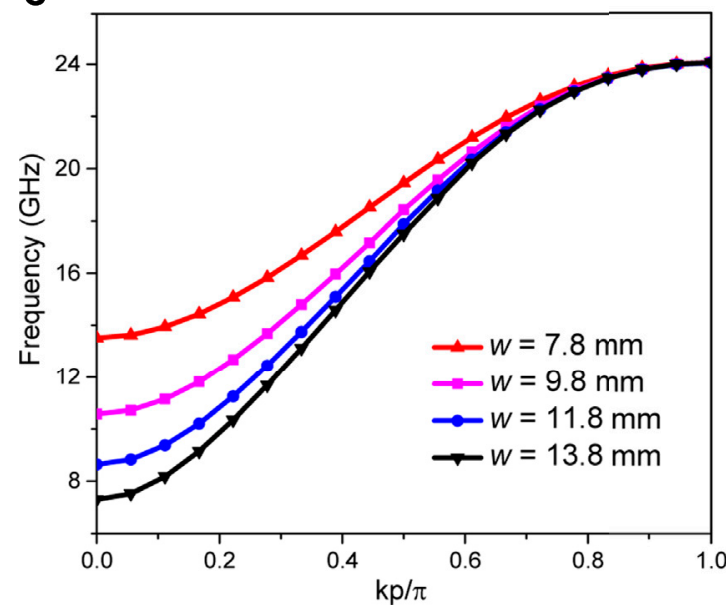

FIGURE 1 | (A) Schematic configuration of the proposed SSPP unit cell and its dispersion curves with different values of (B) parameter $r$ and $(\mathbf{C})$ parameter $w$.

notch filters based on SSPPs have been presented [17-20]. In [19], by changing the voltage of varactor diodes, the center frequency of the notch band can be tuned continuously, but the insertion loss performance is obviously deteriorated after introducing the notch. In [20], a switchable notch filter is proposed whose notch frequency can be changed by switching the states of the PIN diodes, however, the bandwidth of the notch band is very narrow and its selectivity is also not good.

Substrate integrated waveguide (SIW) naturally has the high-pass filtering characteristics, which has been widely applied in the design of microwave devices and antennas [21-24]. In this paper, a new BPF using SSPPs is proposed through loading ring slots and vias within the conventional SIW. Then, four half-wavelength circular slots are added on two sides of the SSPPs located at the center of the circuit to introduce a high-selectivity notch band. In order to make the notch band switchable, four full-wavelength circular slots and four PIN diodes with bias voltages are applied instead of the four half-wavelength circular slots. Two BPF examples with and without switchable notch bands are fabricated and measured for demonstrations.

\section{BPF BASED ON SSPPS WITH A NOTCH BAND}

A new SSPP unit cell based on SIW with a ring slot and a via hole is proposed, as shown in Figure 1A, where it is designed on the dielectric substrate with a relative permittivity of 2.65 , a thickness of $0.5 \mathrm{~mm}$, and a loss tangent of 0.0015 . The via hole connected to the ground of the bottom metal layer is located at the center of the ring slot for each SSPP unit cell. The dispersion characteristics of the SSPP unit cell are analyzed with different radii of the ring slot $r$ and different widths of the SIW $w$ as shown in Figures 1B,C respectively, where the parameters in Figure $\mathbf{1 A}$ set as $w=$ $9.8 \mathrm{~mm}, p=2.6 \mathrm{~mm}, d_{1}=0.5 \mathrm{~mm}, d_{2}=0.85 \mathrm{~mm}, r=1 \mathrm{~mm}$, and $s=0.15 \mathrm{~mm}$ are as the default values.

In Figure 1B, as the parameter $r$ decreases, the right-edge cutoff frequency will be increased, while the left-edge cutoff frequency remains essentially fixed. Additionally, as the SIW width $w$ increases while other parameters remain unchanged, the left-edge cutoff frequency will be reduced but the right-edge cutoff frequency will keep the same as shown in Figure 1C. Therefore, it indicates that the left- and right-edge cutoff frequencies of the passband can be independently adjusted by changing the width of SIW walls and radius of ring slot embedded into the SIW, respectively, when this SSPP unit cell is used for the design of BPF. Consequently, the bandwidth and center frequency of the proposed SSPP-based BPF will be easily controlled by changing the geometric parameters of the SSPP unit cell.

Based on the above analysis, a corresponding BPF using the proposed SSPPs is designed. It consists of the following parts: microstrip lines as the input and output ports for connecting with the SMA connectors to measure the filter performance, a transition part by using a trapezoidal microstrip line and a smaller ring slot for the transition from microstrip line to SSPP waveguide, and a SSPP waveguide with seven periodic unit cells. The width of the microstrip line is set as $w_{0}=1.35 \mathrm{~mm}$ for realizing $50-\Omega$ characteristic impedance. Then, four half-wavelength circular slots are added on two sides of the SSPP located at the center of the circuit to introduce a high-selectivity notch band. Figure 2 shows the schematic configuration of the BPF based on SSPPs with the high-selectivity notch band, where all parametric dimensions are listed in Table 1.

In order to further quantitatively evaluate the performance of the BPF using the proposed SSPP, a prototype is fabricated as shown in Figure 3A with the same parameters in Table 1. The SMA connectors are used at the input and output ports for measurement. Figure 3B shows the comparisons between the 

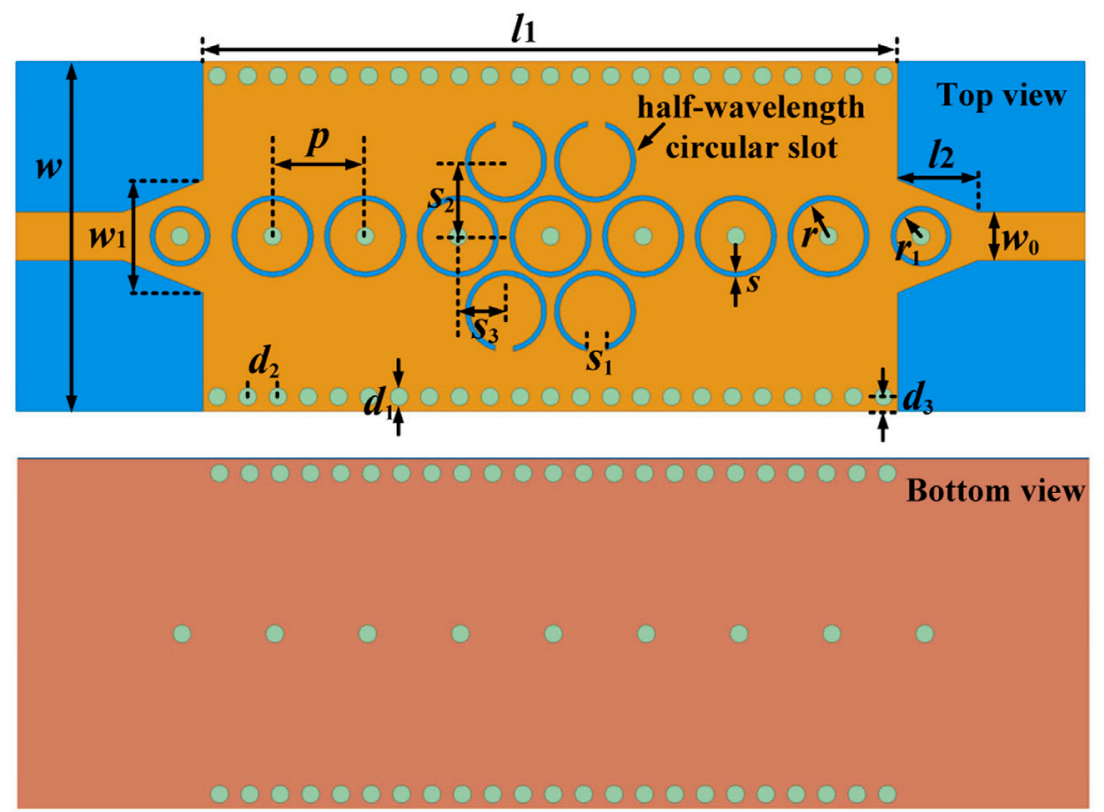

FIGURE 2 | Schematic configuration of the BPF with a high-selectivity notch band.

TABLE 1 | Parameters of the Proposed BPF with A Notch Band.

\begin{tabular}{lcccccccc} 
Parameters & $\boldsymbol{w}$ & $\boldsymbol{w}_{\mathbf{0}}$ & $\boldsymbol{w}_{\mathbf{1}}$ & $\boldsymbol{d}_{\mathbf{1}}$ & $\boldsymbol{d}_{\mathbf{2}}$ & $\boldsymbol{d}_{\mathbf{3}}$ & $\boldsymbol{I}_{\mathbf{1}}$ & $\boldsymbol{I}_{\mathbf{2}}$ \\
\hline Values $(\mathrm{mm})$ & 9.8 & 1.35 & 3.15 & 0.5 & 0.85 & 0.4 & 19.5 & 2.25 \\
Parameters & $p$ & $r$ & $r_{1}$ & $s$ & $s_{1}$ & $s_{2}$ & $s_{3}$ & \\
Values $(\mathrm{mm})$ & 2.6 & 1 & 0.7 & 0.15 & 0.5 & 2.1 & 1.3 & \\
\hline
\end{tabular}

simulated and measured $S$-parameters, where the left- and rightedges of the passband are at 11.3 and $24.1 \mathrm{GHz}$, respectively, basically consistent with the cutoff frequencies in Figure 1 for the parameters set in Table 1. Good agreement between the simulation and measurement of the $S$-parameters can be observed, where the center frequency is located at $17.7 \mathrm{GHz}$ and the relative bandwidth is $72.3 \%$. Slight discrepancies between the measured and simulated results are mainly due to the tolerance of manufacturing and manual soldering for connectors. The insertion loss and return loss within the passband but excluding the notch band are better than 1.7 and $11 \mathrm{~dB}$, respectively. The notch band introduced by the halfwavelength circular slot resonators has high-selectivity characteristics with the center frequency at $16.7 \mathrm{GHz}$ and bandwidth from 16.25 to $17.15 \mathrm{GHz}$. The rejection within the notch band can be up to $19.6 \mathrm{~dB}$. Furthermore, the working frequency of the notch band can be moved if the lengths of the half-wavelength circular slot resonators are changed.

To verify the propagation characteristics of the SSPP waveguide with the notch band, the electric field distributions at three different frequency points located within the notch band and the passband, and at the out of the passband are illustrated in Figures 4A,B,C, respectively. For the notch frequency at $16.8 \mathrm{GHz}$ in Figure $\mathbf{4 A}$, the electric fields are mainly

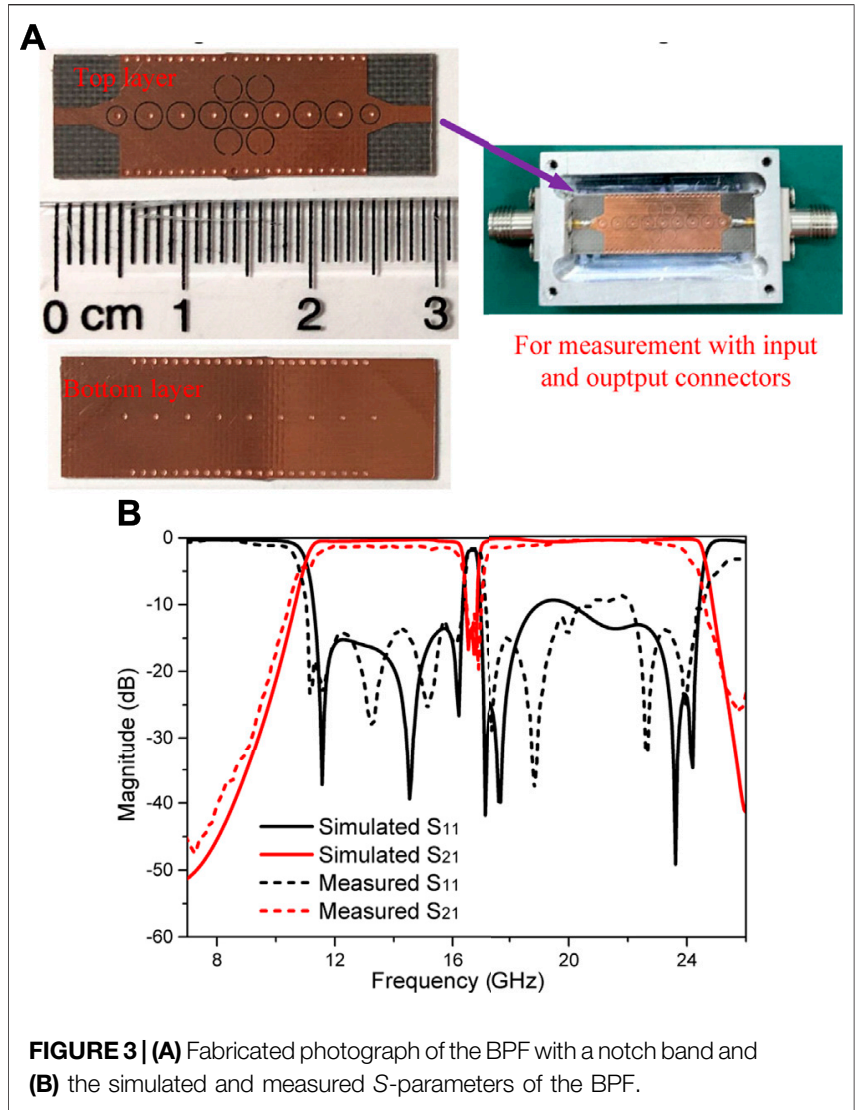

concentrated around the four half-wavelength circular slot resonators on both sides, and the electromagnetic waves disappear after going through the resonators. It indicates that 
A

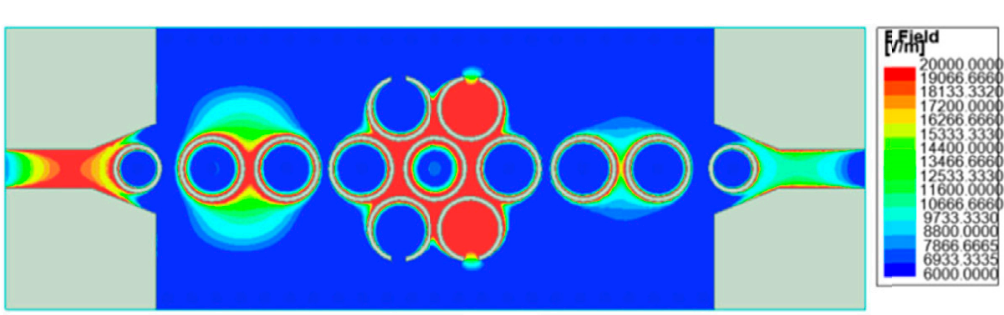

B

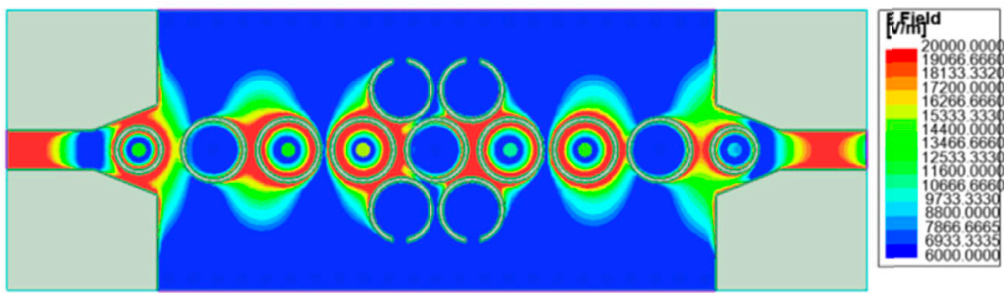

C

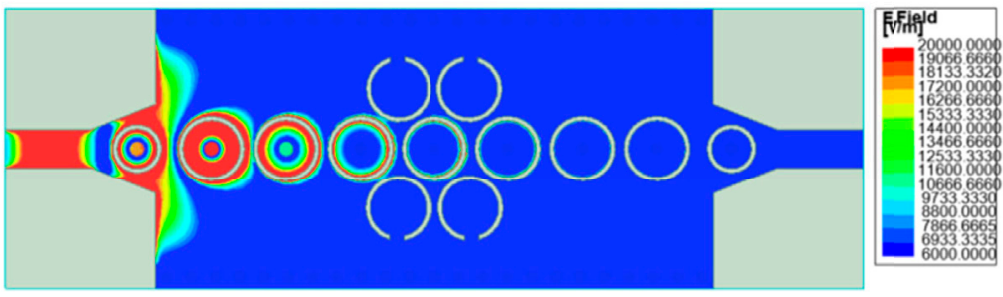

FIGURE 4 | Simulated electric field distributions of the BPF based on the proposed SSPP at (A) $16.8 \mathrm{GHz}$, (B) $22 \mathrm{GHz}$, and (C) $26 \mathrm{GHz}$.

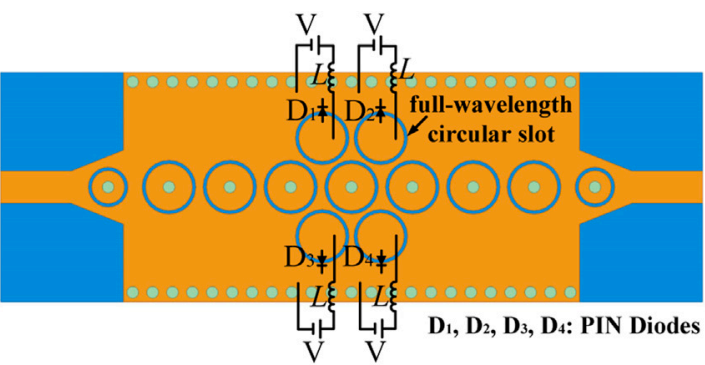

FIGURE 5 | Schematic configuration of the proposed SSPP-based BPF with the switchable notch band.

the four resonators play the role of generating the notch band to reject the signal propagation. In Figure 4B, the SSPP waves can propagate efficiently through the waveguide at $22 \mathrm{GHz}$. In contrast, the SSPP waves decay quickly at the out-of-band, such as at $26 \mathrm{GHz}$ in Figure 4C.

\section{BPF BASED ON SSPPS WITH A SWITCHABLE NOTCH BAND}

In order to make the notch band switchable, four closed-loop ring slot resonators and four PIN diodes are applied instead of the four half-wavelength circular slots, as illustrated in Figure 5. It is well

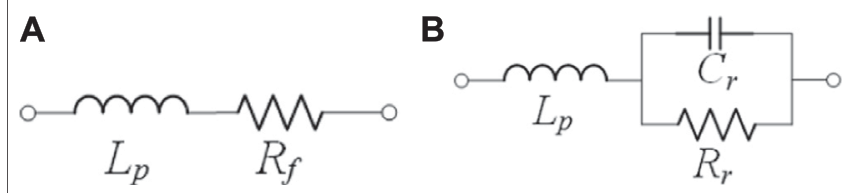

FIGURE 6 | Equivalent circuit models of the PIN diode (A) under the ON state and (B) under the OFF state.

known that a closed-loop ring slot resonator operates at the resonant frequency with one wavelength, therefore, herein it is called as the full-wavelength circular slot. The resonant frequency of the half-wavelength circular slot resonator is just about a half of the full-wavelength circular slot resonator. As the PIN diodes are under the ON state, the closed-loop ring slot resonators will be equivalent to the halfwavelength circular slots as shown in Figure 2, and the notch band will be generated within the passband of BPF. On the contrary, as the PIN diodes are under the OFF state, the notch band will disappear since the resonance generated from the full-wavelength circular slots will be excited at the out-of-band without influence on the passband.

The PIN diodes BAR64-02v (from Infineon) with sizes of $1.6 \mathrm{~mm} \times 0.8 \mathrm{~mm}$ are used and modeled based on the equivalent circuit models shown in Figure 6. As the PIN diode is under the $\mathrm{ON}$ state, the equivalent model is a series circuit of an inductance 


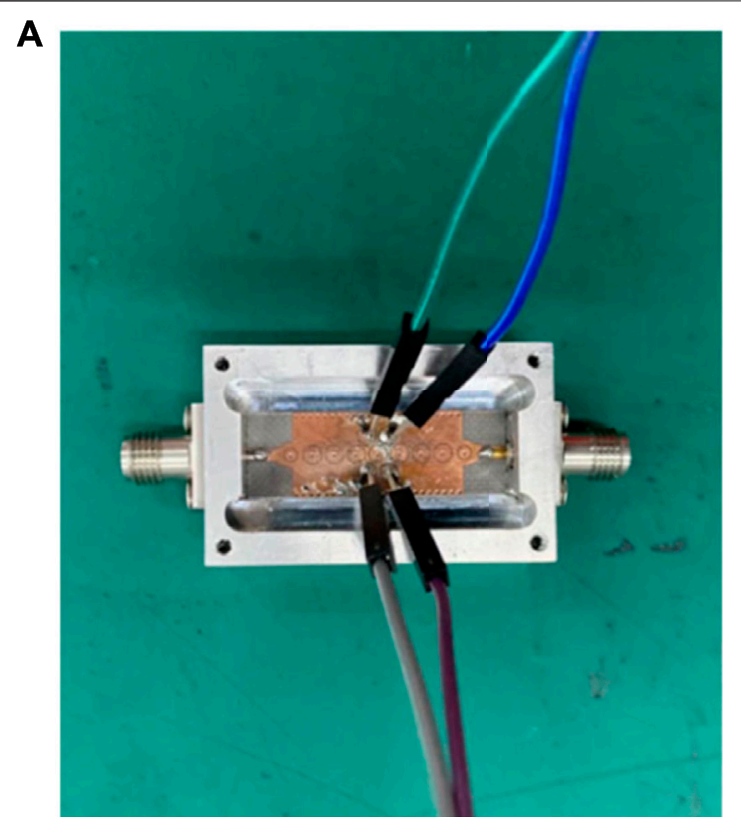

B

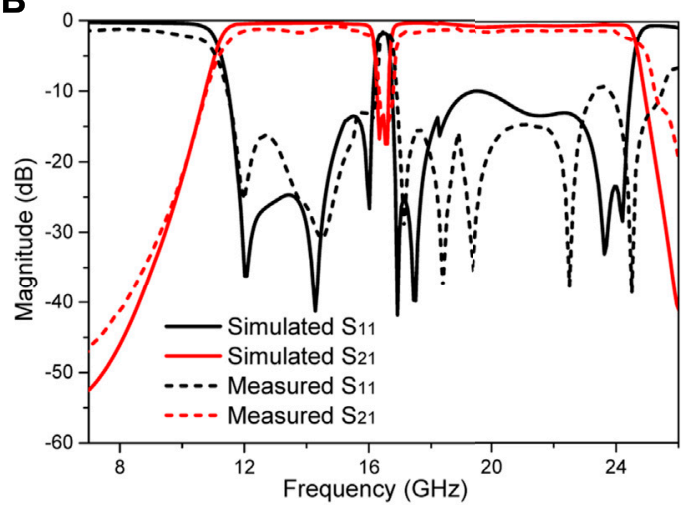

C

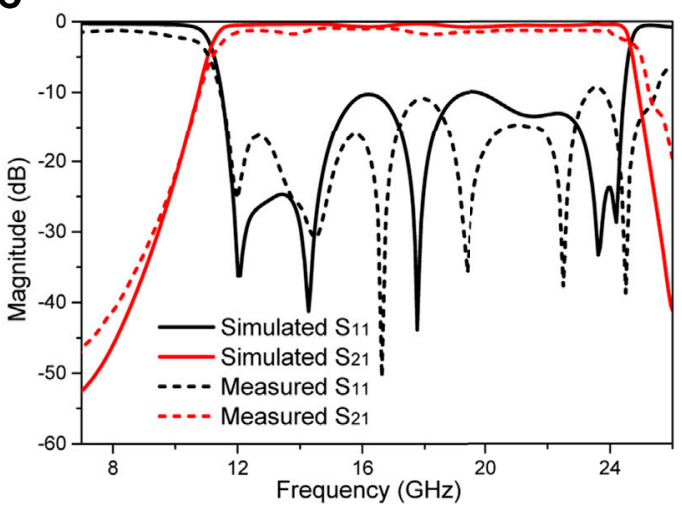

FIGURE 7 | (A) Fabricated prototype of the proposed BPF with a switchable notch band, and its simulated and measured $S$-parameters when the PIN diodes are under the (B) ON state and (C) OFF state.

$L_{p}=0.4 \mathrm{nH}$ and a small resistance $R_{f}=2.4 \Omega$. As the PIN diode is under the OFF state, it can be equalized as an inductance $L_{p}$ in series with a parallel circuit of a small capacitance $C_{r}=0.12 \mathrm{pF}$ and a large resistance $R_{r}=3,000 \Omega$. The four PIN diodes are bridged across the full-wavelength circular slot resonators as seen in Figure 5. The simple DC biasing circuit (bias voltage $\mathrm{V}=1 \mathrm{~V}$ ) with choke inductors $(L=220 \mathrm{nH})$ has little influence on the transmission performance of the filter. Therefore, the proposed BPF based on SSPPs has the switchable function of the notch band.

For demonstration, the proposed BPF based on SSPPs with a switchable notch band is fabricated and measured. Figure 7A shows the fabricated prototype of the proposed BPF and its simulated and measured $S$-parameters under the ON and OFF states of PIN diodes are shown in Figures $\mathbf{7 B}, \mathbf{C}$, respectively. The simulations and measurements are in reasonably good agreement, and the center frequency of the notch band is at $16.5 \mathrm{GHz}$ when the PIN diodes are under the ON state. Compared with the results in Figure 3, the notch band has a slight redshift, which is probably due to the effective length of the half-wavelength circular slot resonator under the ON state of the switchable case larger than that of the fixed case in Figure 3.

\section{CONCLUSION}

In this paper, two BPFs based on the proposed SSPPs with a fixed notch band and a switchable notch band are presented. Four PIN diodes are bridged across the full-wavelength circular slot resonators to construct two switchable states, i.e., ON state and OFF state. The resonators behave as the half-wavelength resonators and full-wavelength resonators by switching the states of diodes. The measured results of the two BPFs are basically in good agreement with the simulated ones. Therefore, the presented works are of greatly promising potentials on the microwave switchable and adaptive devices and circuits.

\section{DATA AVAILABILITY STATEMENT}

The raw data supporting the conclusion of this article will be made available by the authors, without undue reservation.

\section{AUTHOR CONTRIBUTIONS}

All authors listed have made a substantial, direct, and intellectual contribution to the work and approved it for publication.

\section{FUNDING}

This work was supported in part by the NSAF Joint Fund under Grant U2130102 the Scientific Research Projects of Fire Rescue Bureau of Emergency Management Department under Grant 2019XFGG11, in part by the Natural Science Foundation of Shaanxi Province under Grant 2021JQ-060, and in part by the "Siyuan Scholar" Fellowship of XJTU. 


\section{REFERENCES}

1. Barnes WL, Dereux A, and Ebbesen TW. Surface Plasmon Subwavelength Optics. Nature (2003) 424(6950):824-30. doi:10.1038/nature01937

2. Pendry JB, Martín-Moreno L, and Garcia-Vidal FJ. Mimicking Surface Plasmons With Structured Surfaces. Science (2004) 305(5685):847-8. doi:10.1126/science.1098999

3. Shen X, Cui TJ, Martin-Cano D, and Garcia-Vidal FJ. Conformal Surface Plasmons Propagating on Ultrathin and Flexible Films. Proc Natl Acad Sci (2013) 110(1):40-5. doi:10.1073/pnas.1210417110

4. Qi C, Liao S, and Xue Q. Frequency Splitter Based on Spoof Surface Plasmon Polariton Transmission Lines. Appl Phys Lett (2018) 113:161902. doi:10.1063/ 1.5043282

5. Zhao L, Zhang X, Wang J, Yu W, Li J, Su H, et al. A Novel Broadband BandPass Filter Based on Spoof Surface Plasmon Polaritons. Sci Rep (2016) 6:36069. doi:10.1038/srep36069

6. Xu K-D, Zhang F, Guo Y, Ye L, and Liu Y. Spoof Surface Plasmon Polaritons Based on Balanced Coplanar Stripline Waveguides. IEEE Photon Technol Lett (2020) 32(1):55-8. doi:10.1109/lpt.2019.2957059

7. Wu Y, Li M, Yan G, Deng L, Liu Y, and Ghassemlooy Z. Single-Conductor CoPlanar Quasi-Symmetry Unequal Power Divider Based on Spoof Surface Plasmon Polaritons of bow-tie Cells. AIP Adv (2016) 6(10):105110. doi:10.1063/1.4966051

8. Xu KD, Lu S, Guo YJ, and Chen Q. High-Order Mode of Spoof Surface Plasmon Polaritons and its Application in Bandpass Filters. IEEE Trans Plasma Sci (2021) 70(49):269-75. doi:10.1109/tps.2020.3043889

9. Guo YJ, Xu KD, Liu Y, and Tang X. Novel Surface Plasmon Polariton Waveguides With Enhanced Field Confinement for Microwave-Frequency Ultra-Wideband Bandpass Filters. IEEE Access (2018) 6:10249-56. doi:10.1109/access.2018.2808335

10. Kianinejad A, Chen ZN, and Qiu C-W. A Single-Layered Spoof-PlasmonMode Leaky Wave Antenna With Consistent Gain. IEEE Trans Antennas Propagat (2017) 65(2):681-7. doi:10.1109/tap.2016.2633161

11. Zhang X-F, Fan J, and Chen J-X. High Gain and High-Efficiency MillimeterWave Antenna Based on Spoof Surface Plasmon Polaritons. IEEE Trans Antennas Propagat (2019) 67(1):687-91. doi:10.1109/tap.2018.2879847

12. Xu K-D, Guo YJ, and Deng X. Terahertz Broadband Spoof Surface Plasmon Polaritons Using High-Order Mode Developed From Ultra-compact SplitRing Grooves. Opt Express (2019) 27(4):4354-63. doi:10.1364/oe.27.004354

13. Li J, Shi J, Xu K-D, Guo Y-J, Zhang A, and Chen Q. Spoof Surface Plasmon Polaritons Developed From Coplanar Waveguides in Microwave Frequencies. IEEE Photon Technol Lett (2020) 32(22):1431-4. doi:10.1109/lpt.2020.3031065

14. Huan Yi H, Shi-Wei Qu SW, and Xue Bai X. Antenna Array Excited by Spoof Planar Plasmonic Waveguide. Antennas Wirel Propag Lett (2014) 13:1227-30. doi:10.1109/lawp.2014.2332257

15. Guo Y-J, Xu K-D, Deng X, Cheng X, and Chen Q. Millimeter-Wave On-Chip Bandpass Filter Based on Spoof Surface Plasmon Polaritons. IEEE Electron Device Lett (2020) 41(8):1165-8. doi:10.1109/led.2020.3003804

16. Xu K-D, Guo Y-J, Yang Q, Zhang Y-L, Deng X, Zhang A, et al. On-chip GaAsBased Spoof Surface Plasmon Polaritons at Millimeter-Wave Regime. IEEE Photon Technol Lett (2021) 33(5):255-8. doi:10.1109/lpt.2021.3054962
17. Pan BC, Liao Z, Zhao J, and Cui TJ. Controlling Rejections of Spoof Surface Plasmon Polaritons Using Metamaterial Particles. Opt Express (2014) 22(11): 13940-50. doi:10.1364/oe.22.013940

18. Xu J, Zhang HC, Tang W, Guo J, Qian C, and Li WY. TransmissionSpectrum-Controllable Spoof Surface Plasmon Polaritons Using Tunable Metamaterial Particles. Appl Phys Lett (2016) 108(19):824-48. doi:10.1063/1.4950701

19. Zhou YJ, and Xiao QX. Electronically Controlled Rejections of Spoof Surface Plasmons Polaritons. J Appl. Phys (2017) 121(12):123109. doi:10.1063/ 1.4979206

20. Ye L, Chen Y, Wang Z, Zhu C, Zhuo J, and Liu QH. Compact Spoof Surface Plasmon Polariton Waveguides and Notch Filters Based on Meander-Strip Units. IEEE Photon Technol Lett (2021) 33(3):135-8. doi:10.1109/ lpt.2020.3046837

21. Wang Y, Hong W, Dong Y, Liu B, Tang HJ, Chen J, et al. Half Mode Substrate Integrated Waveguide (HMSIW) Bandpass Filter. IEEE Microw Wireless Compon Lett (2007) 17(4):265-7. doi:10.1109/ lmwc. 2007.892958

22. Li W, Xu KD, Tang X, Yang Y, Liu Y, and Liu QH. Substrate Integrated Waveguide Cavity-Backed Slot Array Antenna Using High-Order Radiation Modes for Dual-Band Applications in \$K\$ -Band. IEEE Trans Antennas Propagat (2017) 65(9):4556-65. doi:10.1109/ tap.2017.2723089

23. Guan D, Peng Y, and Zhang Q. Hybrid Spoof Surface Plasmon Polariton and Substrate Integrated Waveguide Transmission Line and its Application in Filter. IEEE Trans Microw Theor Techn (2017) 65:1-8. doi:10.1109/ tmtt.2017.2727486

24. Xu K-D, Xia S, Guo Y-J, Cui J, Zhang A, and Chen Q. W-Band E-Plane Waveguide Bandpass Filter Based on Meander Ring Resonator. IEEE Microw Wireless Compon Lett (2021) 31:1. doi:10.1109/ LMWC.2021.3103638

Conflict of Interest: Author QW is employed by the company Nanjing Ray-Chip Microsystem Co. Limited.

The remaining authors declare that the research was conducted in the absence of any commercial or financial relationships that could be construed as a potential conflict of interest.

Publisher's Note: All claims expressed in this article are solely those of the authors and do not necessarily represent those of their affiliated organizations, or those of the publisher, the editors and the reviewers. Any product that may be evaluated in this article, or claim that may be made by its manufacturer, is not guaranteed or endorsed by the publisher.

Copyright (c) 2021 Tan, Wang, Guo, Cui and Xu. This is an open-access article distributed under the terms of the Creative Commons Attribution License (CC BY). The use, distribution or reproduction in other forums is permitted, provided the original author(s) and the copyright owner(s) are credited and that the original publication in this journal is cited, in accordance with accepted academic practice. No use, distribution or reproduction is permitted which does not comply with these terms. 